\title{
Activation of miR-34a impairs autophagic flux and promotes cochlear cell death via repressing ATG9A: implications for age-related hearing loss
}

\author{
Jiaqi Pang ${ }^{1,2,3,5}$, Hao Xiong ${ }^{1,2,3,5}$, Peiliang Lin ${ }^{1,2}$, Lan Lai ${ }^{1,2,3}$, Haidi Yang ${ }^{1,2}$, Yimin Liu ${ }^{4}$, Qiuhong Huang ${ }^{1,2}$, Suijun Chen ${ }^{1,2}$, Yongyi Ye ${ }^{1,2,3}$, \\ Yingfeng Sun ${ }^{1,2}$ and Yiqing Zheng ${ }^{*, 1,2}$
}

Age-related hearing loss is a major unresolved public health problem. We have previously elucidated that the activation of cochlear miR-34a is correlated with age-related hearing loss in C57BL/6 mice. A growing body of evidence points that aberrant autophagy promotes cell death during the development of multiple age-related diseases. The aim of this study was to investigate the role of miR-34a-involved disorder of autophagy in the pathogenesis of age-related hearing loss. Our results showed that miR-34a expression was markedly upregulated in the aging cochlea accompanied with impairment of autophagic flux. In the inner ear HEIOC1 cell line, miR-34a overexpression resulted in an accumulation of phagophores and impaired autophagosome-lysosome fusion, and led to cell death subsequently. Notably, autophagy-related protein 9A (ATG9A), an autophagy protein, was significantly decreased after miR-34a overexpression. Knockdown of ATG9A inhibited autophagy flux, which is similar to the effects of miR-34a overexpression. Moreover, ursodeoxycholic acid significantly rescued miR-34a-induced HEI-OC1 cell death by restoring autophagy activity. Collectively, these findings increase our understanding of the biological effects of miR-34a in the development of age-related hearing loss and highlight miR-34a as a promising therapeutic target for its treatment.

Cell Death and Disease (2017) 8, e3079; doi:10.1038/cddis.2017.462; published online 5 October 2017

Age-related hearing loss ( $\mathrm{AHL})$, known as presbycusis, is a complex degenerative disease, afflicting $\sim 50 \%$ of people by age 65 years and older. ${ }^{1,2}$ Hearing loss can impair everyday communication in the elderly, causing loneliness and isolation ${ }^{3}$ that make $\mathrm{AHL}$ a risk factor for Alzheimer's disease, dementia, depression and other neuropsychiatric diseases. $^{3-5}$ The detailed mechanisms underlying AHL remain largely unknown. $\mathrm{AHL}$ is a reflection of genetic predisposition as well as a lifetime of insults to the ear, such as the accumulation of noise exposure, ototoxic drugs and diseases. ${ }^{6,7}$ Cochlear degeneration is a common feature in $\mathrm{AHL}$ both in humans and animal models, ${ }^{8}$ including hair cell loss, stria vascularis and spiral ganglion neurons degeneration. $^{3,9}$ The loss of cochlear hair cell is well documented and considered as one of the major causes for AHL. ${ }^{10-13}$ Because hair cells do not regenerate in mammals, clarifying the mechanism of hair cell death is regarded as an important strategy for the treatment of AHL.

Autophagy, a lysosome degradation pathway by which cells capture and deliver damaged proteins and cellular organelles, plays an important homeostatic role in keeping the metabolic balance between synthesis and degradation in stressful environment. ${ }^{14,15}$ Macroautophagy is one of the classical form and the most studied type of autophagy. Briefly, the processes consist two stages: the early stage includes the induction and formation of the phagophore, the formation of the autophagosome, which captures damaged cellular proteins and organelles; the late stage is autophagosomelysosome fusion, which is responsible for degradation and recycling. ${ }^{16,17}$ Recently, aberrant autophagy has been implicated in a variety of neurodegenerative disorders, cancers and autoimmune diseases. ${ }^{17-20}$ Aberrant autophagy makes the clearance of the misfolding and aggregated proteins and organelles ineffectively, contributing to many neurodegenerative disorders, such as Alzheimer's disease, Parkinson's disease and Huntington's disease. ${ }^{18,19,21-23}$ However, the role of autophagy in hearing development, maintenance and multiple types of hearing loss remains to be elucidated. Previous research revealed autophagy attenuates cisplatin and noise-induced hearing loss, ${ }^{24-26}$ while increased autophagic stress may account for premature AHL in SAMP8 mice. $^{27}$

MicroRNAs (miRNAs or miRs) are endogenous, small (20$24 \mathrm{nt}$ ), non-coding RNAs that post-transcriptionally regulate messenger RNA (mRNA) stability and ultimate translation. Growing studies proved that miRNAs regulate aging in worms (Caenorhabditis elegans), mice and humans. ${ }^{28,29}$ Recently, miRNAs were observed in the cochlea and suggested to play an important role in the cochlear pathology. ${ }^{11,30}$ Of interest is miR-34a, which has been implicated as a prime candidate inducing senescence, cell cycle arrest, autophagy and cell death. ${ }^{31,32}$ Our previous study has confirmed that miR-34a is

\footnotetext{
${ }^{1}$ Department of Otolaryngology, Sun Yat-sen Memorial Hospital, Sun Yat-sen University, Guangzhou, China; ${ }^{2}$ Institute of Hearing and Speech-Language Science, Sun Yatsen University, Guangzhou, China; ${ }^{3}$ Guangdong Provincial Key Laboratory of Malignant Tumor Epigenetics and Gene Regulation, Medical Research Center, Sun Yat-sen Memorial Hospital, Sun Yat-sen University, Guangzhou, China and ${ }^{4}$ Guangzhou Occupational Disease Prevention and Treatment Center, Guangzhou, China

${ }^{*}$ Corresponding author: Y Zheng, Department of Otolaryngology, Sun Yat-sen Memorial Hospital and Institute of Hearing and Speech-Language Science, Sun Yat-sen University, 107 West Yan Jiang Road, Guangzhou 510120, China. Tel: +86 20 81332566; Fax: +86 20 81332115; E-mail: yiqingzheng@ @otmail.com

${ }^{5}$ These authors contributed equally to this work.

Received 26.2.17; revised 27.7.17; accepted 02.8.17; Edited by B Zhivotovsky
} 
linked to $A H L,{ }^{11}$ but the detailed mechanism is not fully understood. Recently, miR-34a was reported to be pathologically altered and observed as one of the modulators that regulate autophagy in many neurodegenerative diseases. ${ }^{23,33}$

Thus, we hypothesize that miR-34a activation causes cochlear cell death and $\mathrm{AHL}$ via modulating autophagy, and the strategies aimed at inhibiting miR-34a activity may be beneficial in treating $\mathrm{AHL}$ through autophagy restoration. In the present study, cochlear miR-34a and autophagy status were examined in C57BL/6 mice, a mouse model of AHL. Additionally, modulation of autophagy by miR-34a and the protective effect of ursodeoxycholic acid (UDCA), a suppressant of miR-34a, was assessed in HEI-OC1 cells.

\section{Results}

C57BL/6 mice develop hearing loss and cochlear hair cell loss with aging. Auditory brainstem response (ABR) measurement was used to monitor the progression of AHL. The average thresholds in 3-month old mice $(65.3 \pm 3.0 \mathrm{~dB}$ at $4 \mathrm{kHz}, 57.5 \pm 3.6 \mathrm{~dB}$ at $8 \mathrm{kHz}, 66.5 \pm 2.5 \mathrm{~dB}$ at $16 \mathrm{kHz}$ and $79.8 \pm 1.7 \mathrm{~dB}$ at $32 \mathrm{kHz}$ ) were significantly elevated compared with those in 1-month-old mice $(45.3 \pm 2.8 \mathrm{~dB}$ at $4 \mathrm{kHz}, \quad 31.5 \pm 2.5 \mathrm{~dB}$ at $8 \mathrm{kHz}, \quad 34.8 \pm 2.8 \mathrm{~dB}$ at $16 \mathrm{kHz}$ and $67.3 \pm 1.2 \mathrm{~dB}$ at $32 \mathrm{kHz}$ ). The average thresholds in 12 -month-old mice $(83.3 \pm 1.9 \mathrm{~dB}$ at $4 \mathrm{kHz}, 85.0 \pm 2.0 \mathrm{~dB}$ at $8 \mathrm{kHz}, 98.8 \pm 0.5 \mathrm{~dB}$ at $16 \mathrm{kHz}$ and $99.3 \pm 0.4 \mathrm{~dB}$ at $32 \mathrm{kHz}$ ) $\left(4 \mathrm{kHz}: \quad \mathrm{F}_{(2,57)}=52.7, \quad P<0.001 ; \quad 8 \mathrm{kHz}: \quad \mathrm{F}_{(2,57)}=92.4\right.$, $P<0.001 ; \quad 16 \mathrm{kHz}: \quad \mathrm{F}_{(2,57)}=221.4, \quad P<0.001 ; \quad 32 \mathrm{kHz}$ : $\left.\mathrm{F}_{(2,57)}=168.3, P<0.001\right)$ were significantly elevated compared with those in 1- and 3-month-old mice, indicating that C57BL/6 mice developed early-onset AHL (Figure 1a). To determine whether the functional deficits corresponded to the extent and the localization of hair cell loss, hair cell counts were proceeded by cochleae surface preparations and calculation after the ABR measurements. There was no inner hair cell $(\mathrm{IHC})$ or outer hair cell $(\mathrm{OHC})$ loss in 1-month-old mice at the apical and basal cochlear turns. In 3-month-old mice, only a minor loss of OHCs was apparent in the basal region. Twelve-month-old animals showed $<15 \%$ loss of OHCs $(85.0 \pm 2.4 \%)$ and a $10 \%$ loss of IHCs $(90.0 \pm 4.3 \%)$ in apical turn (OHCs: $\mathrm{F}_{(2,15)}=7.5, P=0.006$; Figure $\left.1 \mathrm{~b}\right)$, while a $85 \%$ loss of OHCs $(15.0 \pm 3.4 \%)$ and a $40 \%$ loss of $\mathrm{IHCs}$
$(60.0 \pm 6.6 \%)$ in the basal turn (IHCs: $F_{(2,15)}=29.1$, $P<0.001$; OHCs: $F_{(2,15)}=277.5, P<0.001$; Figure 1c).

MiR-34a is upregulated and autophagic flux is impaired in the cochlea of C57BL/6 mice with aging. We sought to determine the correlation of miR-34a and autophagy in the aging cochlea. In this sense, we also examine the mRNA expression of $\angle C 3 B$ and $p 62$ that are correlated with autophagy. The elongation of the edges of the phagophore membrane contains a second ubiquitin-like protein, an ATG8 family member, LC3, which is cleaved by ATG4 to form cellular LC3-I. Then, cellular LC3-I is covalently conjugated to phosphatidylethanolamine on the phagophore membrane, in which it is known as LC3-II. ${ }^{34}$ Therefore, LC3-I/ is specifically associated with phagophore and autophagosome membranes serving as a widely used marker to monitor autophagy levels. Another autophagy marker is p62, which is efficiently degraded upon autophagy induction and serves as an index of autophagic degradation. ${ }^{35}$ The expression of miR-34a, LC3B and $p 62$ mRNA was examined in the different ages of C57BL/6 mice via real-time PCR. The miR-34a expression was significantly upregulated during aging $\left(F_{(2,10)}=83.802, P<0.001\right.$; Figure 2a). Conversely, LC3B mRNA expression was slightly decreased in the cochlea with aging $\left(F_{(2,10)}=1.378, P=0.30\right.$; Figure $\left.2 b\right)$, whereas $p 62$ mRNA expression showed a significant age-related elevation $\left(F_{(2,10)}=60.771, P<0.001\right.$; Figure 2c). Consistent with the findings in mRNA alteration, western blot analysis revealed similar changes in LC3-II and p62 protein in different ages (Figure 2d). LC3-II protein levels in the cochlea were decreased $\left(F_{(2,10)}=30.769, P<0.001\right.$; Figure $\left.2 e\right)$, whereas p62 expression was elevated with aging $\left(F_{(2,10)}=77.077\right.$, $P<0.001$; Figure 2f).

MiR-34a overexpression impairs autophagic flux and induces $\mathrm{HEI}-\mathrm{OC} 1$ cell death. To validate the hypothesis that increased miR-34a is associated with autophagy impairment in aging cochlea, the $\mathrm{HEI}-\mathrm{OC} 1$ cell lines, an extensively used cell line used to elucidate pathways of hair cell pathology, ${ }^{36,37}$ were transfected with a miR-34a mimic or a negative control. Real-time PCR analysis showed that the miR-34a mimic increased $p 62$ mRNA levels without a significative change of LC3B expression (miR-34a: $t_{(4)}=-6.2, P=0.003$ (Figure 3a); LC3B: $t_{(4)}=2.45, P=0.067$ (Figure 3b); p62: $t_{(4)}=-12.8$, $P<0.001$; Figure $3 \mathrm{c}$ ). Western blot findings demonstrated


Figure 1 Hearing loss and cochlear hair cell loss in C57BL/6 mice with aging. (a) Elevated ABR thresholds were observed in C57BL/6 mice at 4, 8, 16 and 32 kHz according to the age. ${ }^{*} P<0.05$ compared with 1 month; ${ }^{\#} P<0.05$ compared with 3 months. $N=20$ per age group. Hair cell counts obtained from two representative cochlear locations, in the apical turn (b) and basal turn (c), at different ages. ${ }^{\star} P<0.05 . N=6$ in each group. Date were represented as the mean \pm S.D. $1 \mathrm{M}$, 1 month old; $3 \mathrm{M}$, 3 month old; $12 \mathrm{M}$, 12 month old 
a

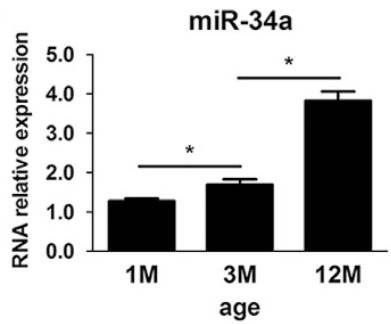

d

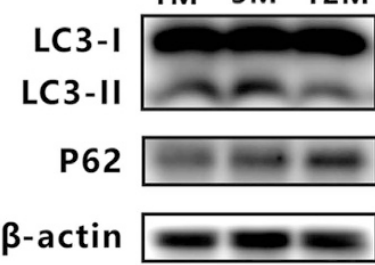

b



e

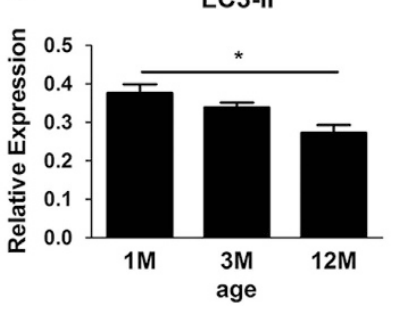

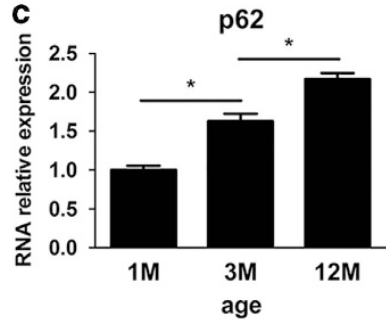

f

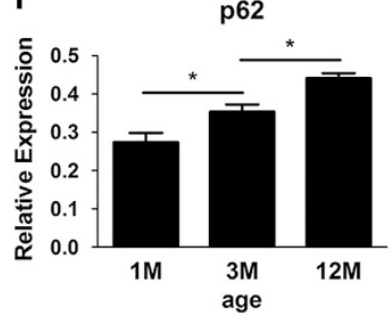

Figure 2 miR-34a activation and the impairment of autophagic flux in the cochlea with aging. (a-c) Quantitative real-time polymerase chain reaction analysis of miR-34a, $L C 3 B$ and $p 62$ in different ages of C57BL/6 mice. Date were represented as the mean \pm S.E.M. obtained from four independent experiments. (d-f) Western blot and densitometry of $L C 3-I /$ and $p 62 .{ }^{*} P<0.05$. Date were represented as the mean \pm S.D. obtained from four independent experiments. $1 \mathrm{M}, 1$ month old; $3 \mathrm{M}, 3$ month old; $12 \mathrm{M}, 12$ month old

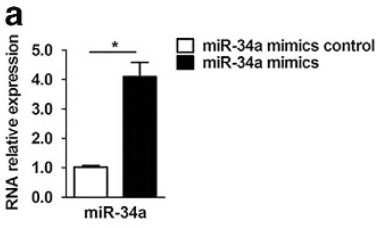

d miR-34a mimics control $0 \quad 20 \quad 40 \quad 0 \quad 0 \quad(n M)$ miR-34a mimics

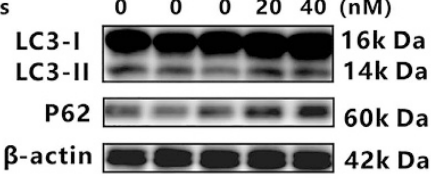

g miR-34a mimics control $024 \mathrm{~h} 48 \mathrm{~h} 72 \mathrm{~h} \quad 0 \quad 0 \quad 0 \quad 0$ miR-34a mimics



$\beta$-actin $\longrightarrow 2 \mathrm{k} \mathrm{Da}$


miR-34a mimics
miR-34a mimics
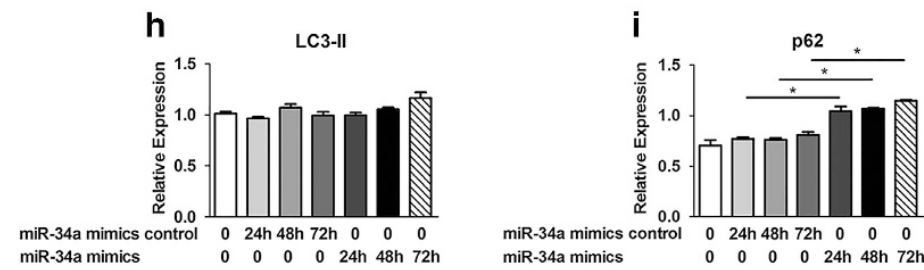

Figure 3 miR-34a modulates autophagy in HEl-OC1 cells. (a-c) Quantitative PCR (qPCR) analysis of miR-34a modulation of LC3B, p62. Date represent the mean \pm S.E.M. obtained from three independent experiments. (d) Western blot of $L C 3-1 /$ and $p 62$ under various treatment with a miR-34a mimic (20 and $40 \mathrm{nM}$ ) or miR-34a mimic control. (e and f) Densitometry analysis of (d). (g) Western blot of LC3-II and p62 under various time after miR-34a mimic transfection. (h and i) Densitometry analysis of (g). ${ }^{*} P<0.05$. Date were represented as the mean \pm S.D. obtained from three independent experiments

that miR-34a overexpression increased $p 62$ protein in a dose-dependent manner (p62 expression in miR-34a mimics $20 \mathrm{nM}: \quad t(4)=-7.6, \quad P=0.002 ; \quad$ in $\quad 40 \mathrm{nM}: \quad t_{(4)}=-14.1$, $P<0.001$; Figures $3 d-f)$ and a time-dependent manner (p62 expression at $24 \mathrm{~h}$ after miR-34a mimics transfection: $t_{(4)}=-10.2, P=0.001$; at 48h: $t_{(4)}=-28.4, P<0.001$; at $72 \mathrm{~h}$ : $t_{(4)}=-17.6, P<0.001$; Figures $\left.3 g-i\right)$. These findings suggested that miR-34a may impair autophagic flux.

To determine whether miR-34a could inhibit actual autophagic flux in HEI-OC1 cells, mRFP-GFP-LC3 adenoviral vectors were used to evaluate the autophagic level treated with miR-34a mimic or inhibitor. In general, LC3 appears as a diffuse pattern in the cytoplasm. After autophagy is activated, LC3 gathers and appears as a punctate pattern. The GFP signal is sensitive to the acidic conditions of the lysosome lumen, whereas mRFP is more stable. Therefore, the yellow punctum, which is colocalized of both GFP and mRFP fluorescence, indicates a compartment that has not fused with a lysosome, such as the phagophore or an autophagosome, whereas the red punctum from an mRFP signal without 

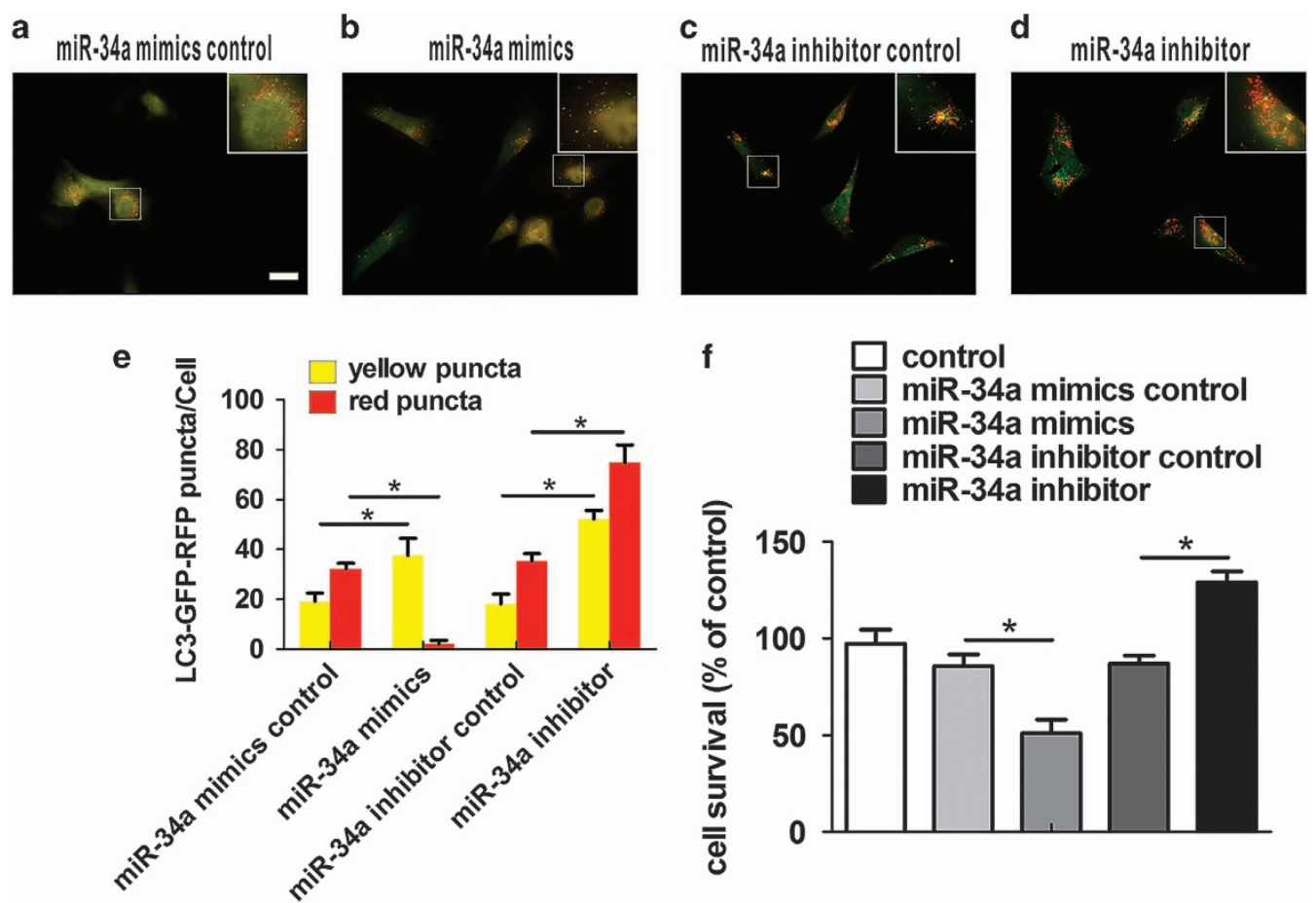

Figure 4 miR-34a promotes HEI-OC1 cells death by impairing autophagic flux. Fluorescence images of mRFP-GFP-LC3 in HEI-OC1 cells treated with a miR-34a mimic control (a), miR-34a mimic (b), miR-34a inhibitor control (c) or miR-34a inhibitor (d). Scale bars: $10 \mu \mathrm{m}$. Quantity analysis of yellow and red puncta was detected (e). Date were represented as the mean \pm S.E.M. obtained from five independent experiments. The MTS assay was performed to examine the viability of $\mathrm{HEI}-\mathrm{OC} 1$ cells under various conditions (f). Date were represented as the mean \pm S.E.M. obtained from three independent experiments. ${ }^{*} P<0.05$

GFP corresponds to an amphisome or autolysosome. Thus, autophagic flux can be determined by evaluating the number of yellow and red puncta. ${ }^{35}$ Generally, both yellow and red puncta were in the cytoplasm (Figures $4 a$ and $c$ ). As expected, HEI-OC1 cells treated with miR-34a mimic only showed accumulation of yellow puncta in the perinuclear region and cytoplasm (miR-34a mimics versus its control: yellow puncta: $t(8)=5.4, P=0.001$; red puncta: $t(8)=-30.3, P<0.001$; Figures $4 \mathrm{~b}$ and $\mathrm{e}$ ), which implied miR-34a prevented the compartment from fusing with a lysosome and blocked autophagosome-lysosome fusion. Conversely, miR-34a inhibition increased both yellow and red puncta, showing the accelerated and unobstructed autophagic flux in HEI-OC1 cells (miR-34a inhibitor versus its control: yellow puncta: $t(8)=14.4, \quad P<0.001$; red puncta: $t(8)=11.3, \quad P<0.001$; Figures $4 \mathrm{~d}$ and $\mathrm{e}$ ). To explore the functional effect of miR-34a on cell survival, cell viability was measured in cells transfected with the miR-34a mimic and miR-34a inhibitor. Compared with the controls, the MTS (3-(4,5-dimethylthiazol2-yl)-5-(3-carboxymethoxyphenyl)-2-(4-sulfophenyl)-2H-tetrazolium) assay showed that $\mathrm{HEI}-\mathrm{OC} 1$ cells transfected with the miR-34a mimic had a reduced survival rate, whereas the miR-34a inhibitor promoted cell survival (miR-34a mimics versus its control: $t_{(4)}=6.4, P=0.003$; miR-34a inhibitor versus its control: $t_{(4)}=-10.6, P<0.001$; Figure $4 f$ ).

MiR-34a regulates autophagy through ATG9A in HEI-OC1 cells. The previous study has demonstrated that $A T G 9 A$ is one of the major targets of $\mathrm{miR}-34 \mathrm{a}^{31}$ and related to the growth of autophagic membranes. ${ }^{35,38}$ To test whether
miR-34a modulates autophagy via ATG9A in HEI-OC1, cells were transfected with a miR-34a mimic or miR-34a inhibitor, with a negative control miRNA mimic or inhibitor. Overexpression of miR-34a in $\mathrm{HEI}-\mathrm{OC} 1$ cells resulted in a marked downregulation of ATG9A expression $\left(t_{(4)}=15.9, P<0.001\right.$; Figures $5 \mathrm{a}$ and b). Oppositely, inhibition of miR-34a increased ATG9A expression $\left(t_{(4)}=27.9, \quad P<0.001\right.$; Figures $5 \mathrm{c}$ and $\mathrm{d}$ ). Next, we sought to determine the effects of ATG9A depletion on autophagy using siRNA-mediated knockdown of ATG9A (Figure 5e). Knockdown of ATG9A resulted in an accumulation of $p 62$ without a significant change of LC3-II expression that was similar to that mediated by miR-34a overexpression ( $L C 3-I I: t_{(4)}=-2.0, P=0.123$; p62: $t_{(4)}=-6.1, P=0.004$; Figures $5 \mathrm{~g}$ and $\mathrm{h}$ ). The Ad-mRFPGFP-LC3 transfection were performed before the si-ATG9A knockdown transfection. Interestingly, $\mathrm{HEI}-\mathrm{OC} 1$ cells treated with si-ATG9A showed a few red puncta and plenty of yellow puncta in the perinuclear region and cytoplasm (yellow puncta: $t_{(4)}=-0.661, P=0.544$; red puncta: $t_{(4)}=13.9$, $P<0.001$; Figures $5 \mathrm{i}-\mathrm{k})$. Knockdown of $A T G 9 A$ also led to the blockage of autophagosome-lysosome fusion as what miR-34a overexpression did, demonstrating the possibility that miR-34a inhibits autophagosome-lysosome fusion by deregulation of ATG9A.

UDCA rescues $\mathrm{HEI}-\mathrm{OC1}$ cells from death through autophagy recovery. UDCA is a potent modulator of miRNA transcription involved in apoptosis, cell cycle control, proliferation and cell growth. ${ }^{39,40}$ As UDCA reduces wild-type miR-34a promoter activity after p53 overexpression and 
a miR-34a mimics control +-
miR-34a mimics
ATG9A $-110 \mathrm{k} \mathrm{Da}$
$\beta$-actin $-42 \mathrm{k} \mathrm{Da}$ b miR-34a mimics control

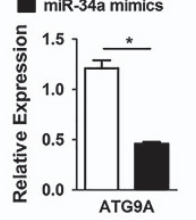

C

miR-34a inhibitor control + miR-34a inhibitor d

$\square$ miR-34a inhibitor control miR-34a inhibitor

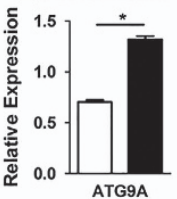

e

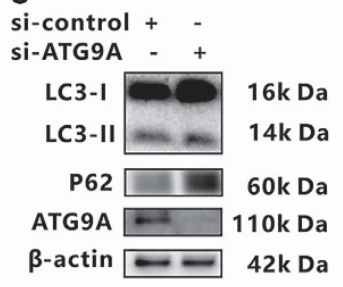

f



g

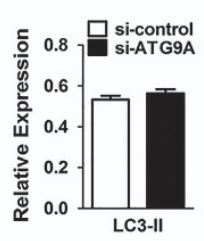

h

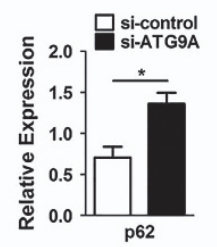

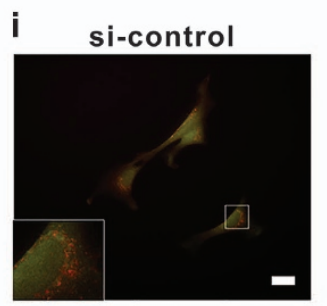
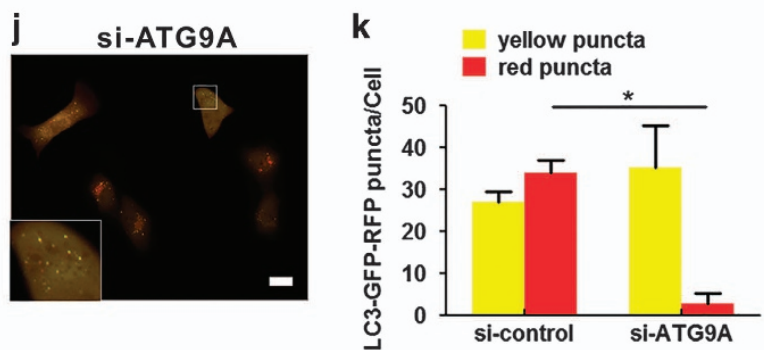

Figure 5 ATG9A is targeted by miR-34a in the regulation of autophagy. (a and $\mathbf{b}) \mathrm{HEl}-\mathrm{OC} 1$ cells transfected with miR-34a mimics and its control were subjected to western blot analysis for ATG9A. (c and d) Western blot and densitometry analysis performed after the transfection of miR-34a inhibitor and inhibitor control. (e-h) HEl-OC1 cells were transfected with si-control and si-ATG9A. Seventy-two hours post-transfection, total protein was harvested and subjected to western blot analysis for ATG9A, LC3-II and p62, and $\beta$-actin was used as a loading control. Quantification of band intensities normalized to $\beta$-actin and relative to control are shown below respective blots. Fluorescence images of mRFP-GFP-LC3 in HEI-OC1 cells treated with a si-control (i) and si-ATG9A (j) with a nutrition-free medium for $6 \mathrm{~h}$ before fixation. Quantity analysis of yellow and red puncta was detected (k). Scale bars: $10 \mu \mathrm{m}$. Date were represented as the mean \pm S.E.M. obtained from four independent experiments. ${ }^{*} P<0.05$



miR-34a mimics control +-++

miR-34a mimics
UDCA

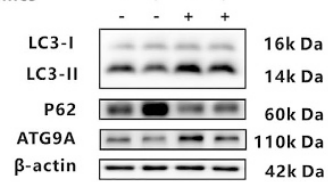

b

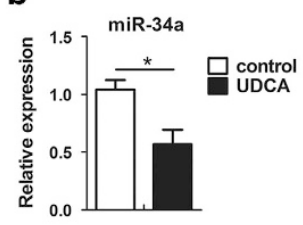

C

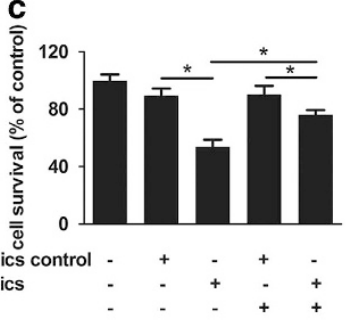
$\begin{array}{llllll}\text { miR-34a mimics control } & - & + & - & + & - \\ \text { miR-34a mimics } & - & - & + & - & + \\ \text { UDCA } & - & - & - & + & +\end{array}$

$\mathbf{f}$
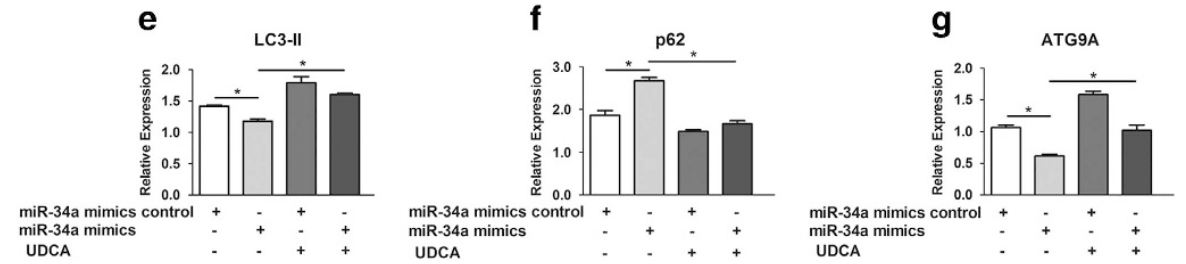

Figure 6 UDCA protects HEI-OC1 cells from miR-34a mimic-induced cell death. (a) Cell survival experiment was detected in various concentration of UDCA. (b) MiR-34a expression was performed after being treated with or without $10 \mu \mathrm{M}$ UDCA for $24 \mathrm{~h}$. (c) The MTS assay was performed to examine the viability of HEI-OC1 cells under miR-34a overexpression with or without UDCA treatment. The HEI-OC1 cell viability assessed via MTS assay. (d-g) Western blot analysis and densitometry of LC3-II, p62 and ATG9A. ${ }^{*} P<0.05$. Date were represented as the mean \pm S.E.M. obtained from three independent experiments

hampers miR-34a expression by almost $40 \%$ in rat liver, ${ }^{39,41}$ we wondered if UDCA treatment reduces miR-34a expression in HEl-OC1 cells. Cell survival experiment showed $10 \mu \mathrm{M}$ UDCA treatment in $24 \mathrm{~h}$ had the best effects of survival, and $200 \mu \mathrm{M}$ was median lethal dose $\left(\mathrm{F}_{(7,16)}=53.7\right.$, $P<0.001$; Figure 6a). Ten micromolar UDCA treatment significantly decreased miR-34a expression $\left(t_{(4)}=5.5\right.$, $P=0.005$; Figure $6 \mathrm{~b})$. The MTS assay results showed that 


\section{Normal autophagic flux}

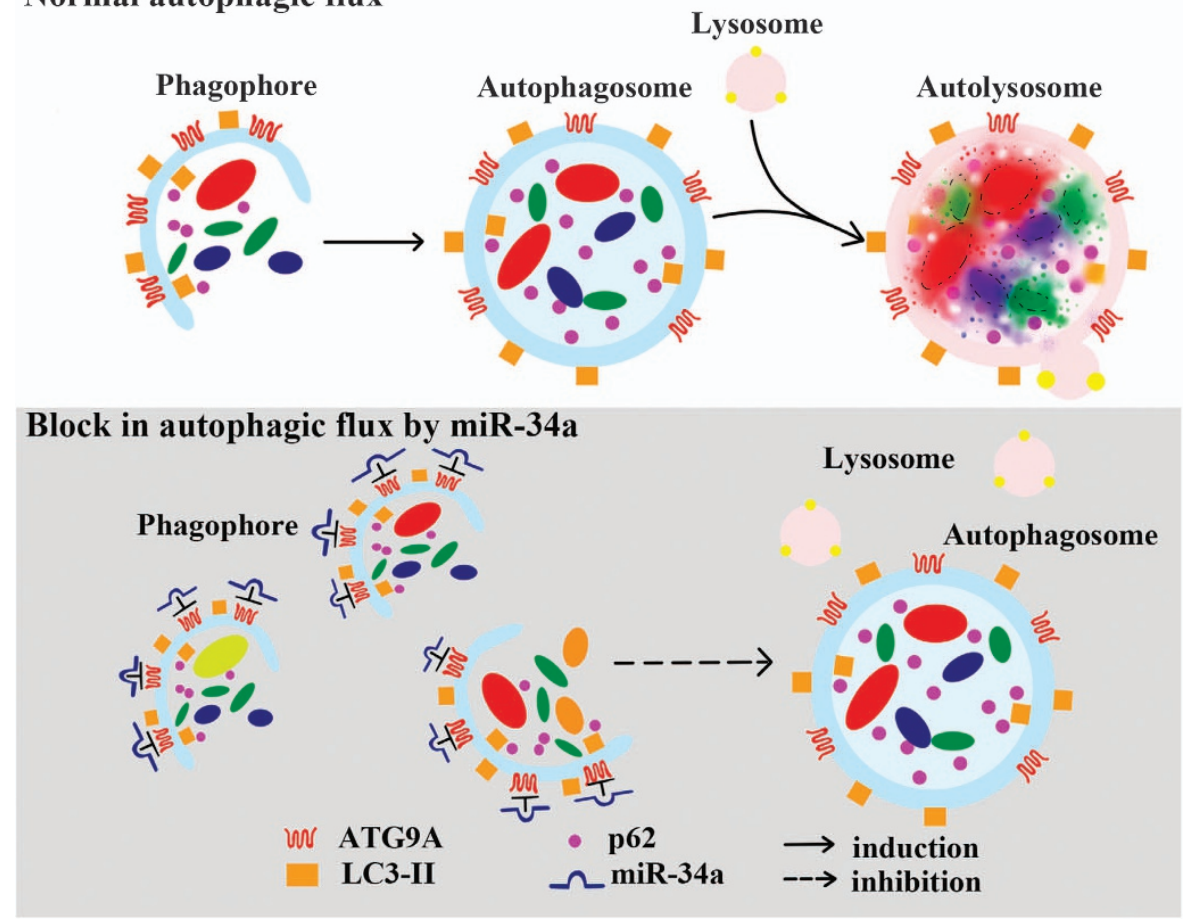

Figure 7 Schematic model demonstrating the elevation of miR-34a impairs autophagic flux through ATG9A. ATG9A, the only multipass transmembrane ATG protein, is required for the expansion of autophagic membranes. Under normal condition, the initiation of autophagy includes the formation of the phagophore, membrane closure to encapsulate contents in the autophagosome. Completion of the autophagosome is followed by fusion with lysosomes and degradation of the contents. We favor the hypothesis that in the case of miR-34a overexpression, miR-34a inhibition of ATG9A impairs autophagosome biogenesis leading to phagophore accumulation. Therefore, the formation of autophagosome is inhibited and the autophagic flux is limited. LC3-II is specifically associated with phagophore and autophagosome membranes serving as a widely used marker to monitor autophagy levels. Another autophagy marker is $p 62$, which is efficiently degraded upon autophagy induction and serves as an index of autophagic degradation

$10 \mu \mathrm{M}$ UDCA treatment significantly reduced miR-34a mimicinduced cell death $\left(\mathrm{F}_{(4,10)}=41.1, P<0.001\right.$; Figure $\left.6 \mathrm{c}\right)$. The western blot analysis indicated that $10 \mu \mathrm{M}$ UDCA treatment significantly restored autophagic flux impairment, by the increase of $p 62$ degradation and the recovery of ATG9A expression ( $L C 3-I l: \mathrm{F}_{(3,8)}=72.4, P<0.001 ; p 62: \mathrm{F}_{(3,8)}=124.2$, $P<0.001$; ATG9A: $\mathrm{F}_{(3,8)}=161.0, P<0.001$; Figures $\left.6 \mathrm{~d}-\mathrm{g}\right)$.

\section{Discussion}

In the present study, we demonstrated that miR-34a was activated in AHL accompanied with the impairment of autophagic flux. In addition, in HEI-OC1 cells, miR-34a inhibited autophagic flux through suppressing autophagy protein ATG9A (Figure 7). Moreover, UDCA treatment protected $\mathrm{HEI}-\mathrm{OC} 1$ cells by inhibition of miR-34a and rescued autophagic flux.

$\mathrm{AHL}$ is a progressive neurodegenerative disorder in the auditory system. ${ }^{1,3}$ One of the main causes of AHL is thought to be the irreversible loss of cochlear hair cells in the inner ear. ${ }^{1,3,13}$ However, the detailed mechanism underlying cochlear hair cell death in AHL remains largely unknown. Because the impairment of autophagic flux is suggested to play an important role in neurodegenerative diseases and aging, we were interested in determining whether aberrant autophagy is involved in cochlear hair cell death and AHL induction. In the present study, we found that miR-34a overexpression increased $p 62$ and impaired the autophagic flux in vitro, which is similar to other aging studies. ${ }^{42-44}$ Meanwhile, miR-34a promoted HEI-OC1 cells death, which is consistent with our previous study. ${ }^{11}$ We consider that the activation of miR-34a increased $p 62$ expression through the impairment of autophagic flux and promoted HEI-OC1 cells death. We also found that miR-34a increase was accompanied by the autophagy impairment in the aging cochlea of C57BL/6 mice. These findings suggested that miR-34a modulation of autophagy might be involved in the pathogenesis of AHL in C57 mice. Taken together, we have reason to believe that miR-34a-induced autophagy impairment is correlated with cochlear hair cell death and might contribute to $\mathrm{AHL}$.

The mechanisms underlying miR-34a blockage of autophagy remains largely unknown. In $C$. elegans, miR-34a modulates lifespan via directly repressing the autophagy gene ATG9A. ${ }^{31}$ In mammalian models, gene ATG9A was proved as one of miR-34a target genes and its inhibition was found in neural stem cell differentiation ${ }^{45}$ and cardiac hypertrophy, ${ }^{46}$ while miR-34a was overexpressing. ATG9A protein is necessary for optimal autophagy. ${ }^{4-49}$ The current hypothesis is that ATG9A delivers lipids, which is required for the expansion of autophagosomes. ${ }^{35,38}$ As the only transmembrane ATG protein, ATG9 was supposed to associate with many other compartments, including recycling endosomes, early endosomes and late endosomes, and it may also be possible that 
ATG9A delivers regulators to the growing phagophore. ${ }^{50}$ In parkin-mediated mitophagy, the relocalization of transcription factor EB, a master regulator of lysosomal biogenesis, required ATG9A. ${ }^{51}$ Whether miR-34a blocked autophagic flux via $A T G 9 A$ in HEI-OC1 cells remains unknown. In our vitro study, overexpression of miR-34a caused a significant decrease of ATG9A expression. Knockdown of ATG9A increased the expression of $p 62$ and blocked autophagosome-lysosome fusion, which is similar to the effect of miR-34a overexpression (Figure 7). These findings suggested that miR-34a modulation of autophagy at least partly through its target ATG9A in HEI-OC1 cells. It should be noted that miR-34a mediates autophagy via multiple targets, such as SIRT1 and BCl-2. ${ }^{11,52-54}$ Future studies are undoubtedly needed to address the relationship between other targets of miR-34a and autophagy in HEl-OC1 cells.

Since increased miR-34a is correlated with $\mathrm{AHL}$ and cochlear hair cell loss in C57BL/6 mice, ${ }^{11}$ manipulation of miR-34a is proposed to be a potential intervention for prevention of AHL. As an effective miR-34a inhibitor, UDCA can be used as a cytoprotective agent to treat non-alcoholic fatty liver disease. ${ }^{41}$ Moreover, UDCA plays an antiapoptotic and anti-inflammatory role and is able to cross the blood-brain barrier; therefore, it was introduced as a novel approach for neurodegenerative diseases. ${ }^{55-57}$ However, the role of UDCA in autophagy modulation is controversial. ${ }^{58,59}$ Our in vitro data showed that UDCA treatment decreased miR-34a levels and attenuated miR-34a-induced $\mathrm{HEI}-\mathrm{OC} 1$ cell death. Also, UDCA alleviated $p 62$ changes induced by miR-34a overexpression, implying that UDCA rescued autophagic flux. These findings indicate that UDCA could protect $\mathrm{HEI}-\mathrm{OC} 1$ cells from cell death by restoring the miR-34a-induced autophagic flux. Further studies are needed to confirm whether UDCA treatment is able to protect cochlear hair cells and delay $\mathrm{AHL}$ in the animal model.

\section{Conclusion}

This study revealed that the impairment of autophagy and the elevation of miR-34a were found in the aging cochlea of C57BL/6 mice, which developed AHL. MiR-34a modulated autophagic flux via ATG9A and determined the fate of HEIOC1 cells. Moreover, UDCA treatment prevented HEI-OC1 cells' death from restoring miR-34a-induced autophagy flux impairment. Our results increase our understanding of miR-34a-mediated autophagy in the control of cochlear hair cells fate and the development of $\mathrm{AHL}$ and propose miR-34a as a promising therapeutic target for the treatment of AHL.

\section{Materials and Methods}

Animals. Sixty C57BL/6 mice (Laboratory Animal Center, Sun Yat-sen University, Guangzhou, China) were divided into three groups: 1 month old; 3 month old and 12 month old. Every group had 20 subjects. Animal care and experimental research were approved by the Animal Research Committee, Sun Yat-sen University and by the Animal Research: Reporting In Vivo Experiments guidelines.

Auditory brainstem response. ABR measurements have been described in the previous study. ${ }^{10}$ The measurements were performed by inserting hypodermic needle electrodes at the vertex (active), below the left ear (reference), as well as the right ear (ground) after being narcotized by a peritoneal injection $(100 \mathrm{mg} / \mathrm{kg}$ ketamine and $10 \mathrm{mg} / \mathrm{kg}$ xylazine mixture). The acoustic signals were generated, and using Tucker-Davis Technologies (TDT System III, Alachua, FL, USA) hardware and software processed the responses. Ten millisecond $(\mathrm{ms})$ tone bursts with a $1 \mathrm{~ms}$ rise or fall time were presented at $4,8,16$ and $32 \mathrm{kHz}$ at a rate of 21.1/s. The average response to 1024 stimuli was gained through reducing the sound intensity at $5 \mathrm{~dB}$ intervals near the threshold. The lowest stimulation decibel level where a positive wave in the evoked-response trace was evident was defined and marked.

Tissue preparation. The narcotized mice were killed after ABR recordings, and the cochleae were wiped off and fixed by steeping into $4 \%$ paraform in phosphate-buffered saline (PBS, pH 7.4) about $0.1 \mathrm{mM}$ all night at $4{ }^{\circ} \mathrm{C}$. After that, the cochleae were put into $4 \%$ sodium ethylenediaminetetraacetic acid for 2 days decalcification. For RNA and protein preparations, cochlear tissues were disassembled by minor tongs, quick frozen in liquid nitrogen and reserved at $-80^{\circ} \mathrm{C}$.

Surface preparations and hair cell counts. After $4 \%$ sodium ethylenediaminetetraacetic acid decalcification, the organ of Corti was microdissected, rinsed in precool PBS, immersed in 1\% Triton X-100 for $15 \mathrm{~min}$ at room temperature, $100 \mu$ l of phalloidin (Life Technology, Carlsbad, CA, USA) containing FITC at $37^{\circ} \mathrm{C}$ in the dark for $120 \mathrm{~min}$, followed by incubated with 4',6-diamidino-2phenylindole) (10 mg/ml; Sigma, St. Louis, MO, USA) for $10 \mathrm{~min}$, and mounted on glass slides in $50 \%$ glycerol. Samples were observed and imaged with an Olympus BX63 microscope (Olympus, Tokyo, Japan). We calculated two positions of the hair cell, at $10-20$ and $65-70 \%$ of the whole cochlear duct distance from the apex, which approximately is in line with the $7-8$ or $32-36 \mathrm{kHz}$ frequency domain. Hair cells were calculated in six mice from each group and were considered to be degenerated if the cell nuclei were devoid.

In vitro cell culture of $\mathrm{HEI}-\mathrm{OC} 1$ cells. $\mathrm{HEI}-\mathrm{OC} 1$ cells (kindly provided by $\mathrm{F}$ Kalinec at the House Ear Institute, Los Angeles, CA, USA) were cultured in Dulbecco's modified Eagle's medium (Gibco-Life Technologies, Carlsbad, CA, USA), supplemented with $10 \%$ fetal bovine serum (Gibco) at $33^{\circ} \mathrm{C}$ under $10 \%$ $\mathrm{CO}_{2}$ (permissive conditions).

Transfection of microRNA mimics, inhibitors and ATG9A siRNA. To examine the effect of miR-34a on autophagy and ATG9A, HEI-OC1 cells were transfected with a miR-34a mimic or a negative control of miRNA mimic (GenePharma, Shanghai, China) at 20 or $40 \mathrm{nM}$ and harvested 24,48 or $72 \mathrm{~h}$ later. In addition, HEl-OC1 cells were transfected with an ATG9A siRNA (Ruibo, Guangzhou, China) and harvested $72 \mathrm{~h}$ later to observe whether miR-34a regulate autophagy via ATG9A. The protective effect of UDCA (Sigma) was tested in $\mathrm{HEI}-\mathrm{OC} 1$ cells under $10 \mu \mathrm{M}$.

Quantitative real-time polymerase chain reaction. According to the manufacturer's protocol, total RNA was isolated using TRIzol Reagent (InvitrogenLife Technologies, Carlsbad, CA, USA), with $1 \mu \mathrm{g}$ of total RNA reverse-transcribed using a ReverTra-Plus-TM Kit (Takara, Osaka, Japan). Primer sequences used for amplifications were as follows: LC3B forward: 5 '-TTATAGAGCGATACAAGGGGGAG $-3^{\prime}$ and reverse: $5^{\prime}$-CGCCGTCTGATTATCTTGATGAG-3'; p62 forward: $5^{\prime}$-GAACTC GCTATAAGTGCAGTGT-3' and reverse: 5'-AGAGAAGCTATCAGAGAGGTGG-3'; glyceraldehyde-3-phosphate dehydrogenase: forward: 5'-TGAACGGGAAGCT CACTGG-3' and reverse: 5'-GCTTCACCACCTTCTTGATGTC-3'. Complementary DNA samples were magnified using SYBR Premix Ex Taq (Takara) and detected with the Roche LightCycler 96 Real-Time PCR System (Roche, Basel, Switzerland). Glyceraldehyde-3-phosphate dehydrogenase was used to be internally piloting for $L C 3 B$ and p62 standardization. For miR-34a expressive assay, total RNA was isolated using TRIzol Reagent as well, with $500 \mathrm{ng}$ of RNA reverse transcribed using special miRNA stem-loop primers and a PrimeScript RT reagent Kit (TaKaRa). According to the manufacturer's instructions,mature miRNA expression was measured with TaKaRa Taq Version 2.0 plus dye (TaKaRa, Osaka, Japan) with miRNA levels normalized to U6 small nuclear RNA expression.

Western blot analysis. Cultured cells and cochlear tissues were symmetrical on ice-cold radioimmunoprecipitation assay lysis buffer (Thermo Fisher Scientific, Pittsburgh, PA, USA) lasted about $30 \mathrm{~min}$ and centrifuged at $12000 \times \mathrm{g}$ at $4{ }^{\circ} \mathrm{C}$ lasted $30 \mathrm{~min}$, and the floating layer were gathered. Protein consistence was decided by using a protein test dye agentia (Bio-Rad, Hercules, CA, USA). Protein samples $(20 \mu \mathrm{g})$ were decomposed via sodium dodecyl sulfate polyacrylamide gel electrophoresis, shifting proteins onto a polyvinylidene fluoride membrane (Merck 
Millipore, Billerica, MA, USA) and blocked with $5 \%$ nonfat dry milk in TBS with $0.1 \%$ Tween 20 (TBS-T). The membranes were incubated with anti-LC3B, anti-p62, or anti-ATG9A (1: 1000; Cell Signaling Technology, Danvers, MA, USA) overnight, washed three times (every $10 \mathrm{~min}$ ) with TBS-T and incubated with a proper secondary antibody $(1: 10000)$ for $1 \mathrm{~h}$. After extensive membrane washing, the bands of immune reactivity were illustrated through Enhanced chemiluminescence (Merck Millipore). Band intensities were measured by densimetric test using $\mathrm{NIH}$ Image $\mathrm{J}(\mathrm{NIH}$, Bethesda, MD, USA), and $\beta$-actin was used as a loading and internally piloting to empower specimen standardization.

Cell viability assay. Cell viability were examined by the MTS assay. HEI-OC1 cells in $100 \mu \mathrm{l}$ of nutrient medium were placed in $20 \mu \mathrm{l}$ of Cell Titer 96 Aqueous One Solution test (Promega, Madison, WI, USA) lasted $2 \mathrm{~h}$ according to the manufacturer's instructions and quantified at an absorbance of $490 \mathrm{~nm}$ using a Wellscan MK3 Microplate Reader (Labsystems Dragon, Helsinki, Finland).

Transfection of cells with fluorescence LC3 adenoviral vectors. The mRFP-GFP-LC3 adenoviral vectors (Ad-mRFP-GFP-LC3) were provided by HanBio Technology Co. Ltd (HanBio, Shanghai, China). Dissection of the autophagic flux process by a novel reporter protein, tandem fluorescent-tagged LC3. The Ad was transfected into HEI-OC1 cells according to the manufacturer's protocol at an $\mathrm{MOI}$ of 100 for $6 \mathrm{~h}$. Observation of autophagic flux was determined after fluorescent staining by evaluating the number of GFP and mRFP puncta (puncta/cell were counted).

Statistical analysis. All experiments were independently repeated at least three times. Student's $t$-test and one-way analysis of variance with Fisher's post hoc test were used for statistical analysis. Values of $P<0.05$ were considered significant.

\section{Conflict of Interest}

The authors declare no conflict of interest.

Acknowledgements. This work was supported by the National Natural Science Foundation of China (81570935 and 81570916), the Guangdong Natural Science Foundation (2015 A030313041), the Guangdong Science and Technology Foundation (2013B021800230), the Guangzhou Science and Technology Foundation (2014Y2-00511) and Yat-sen Scholarship for Young Scientist. We kindly thank Dr Huasong Zhang for the schematic drawing.

\section{Author contributions}

JQP: conception and design, designed and performed the experiments, data analysis and interpretation, and manuscript writing; YQZ and HX: conception and design, manuscript writing, final approval of manuscript; PLL, HDY, LL, YYY, YFS, YML, QHH and SJC: assembly of data and data analysis.

\section{Publisher's Note}

Springer Nature remains neutral with regard to jurisdictional claims in published maps and institutional affiliations.

1. Yamasoba T, Lin FR, Someya S, Kashio A, Sakamoto T, Kondo K. Current concepts in agerelated hearing loss: epidemiology and mechanistic pathways. Hear Res 2013; 303: 30-38.

2. Promoting healthy hearing. Lancet $2013 ; 381: 778$.

3. Kidd IA, Bao J. Recent advances in the study of age-related hearing loss: a mini-review. Gerontology 2012; 58: 490-496.

4. Panza F, Solfrizzi V, Logroscino G. Age-related hearing impairment-a risk factor and frailty marker for dementia and AD. Nat Rev Neurol 2015; 11: 166-175.

5. Gates GA, Mills JH. Presbycusis. Lancet 2005; 366: 1111-1120.

6. Bielefeld EC, Tanaka C, Chen GD, Henderson D. Age-related hearing loss: is it a preventable condition? Hear Res 2010; 264: 98-107.

7. Ohlemiller KK. Mechanisms and genes in human strial presbycusis from animal models. Brain Res 2009; 1277: 70-83.

8. Naldi AM, Belfrage C, Jain N, Wei ET, Martorell BC, Gassmann M et al. Neurona erythropoietin overexpression protects mice against age-related hearing loss (presbycusis). Neurobiol Aging 2015; 36: 3278-3287.

9. Someya S, Yu W, Hallows WC, Xu J, Vann JM, Leeuwenburgh C et al. Sirt3 mediates reduction of oxidative damage and prevention of age-related hearing loss under caloric restriction. Cell 2010; 143: 802-812.

10. Pang J, Xiong H, Yang H, Ou Y, Xu Y, Huang $Q$ et al. Circulating miR-34a levels correlate with age-related hearing loss in mice and humans. Exp Gerontol 2016; 76: 58-67.
11. Xiong H, Pang J, Yang H, Dai M, Liu Y, Ou Y et al. Activation of miR-34a/SIRT1/p53 signaling contributes to cochlear hair cell apoptosis: implications for age-related hearing loss. Neurobiol Aging 2015; 36: 1692-1701.

12. Xiong H, Dai M, Ou Y, Pang J, Yang H, Huang $Q$ et al. SIRT1 expression in the cochlea and auditory cortex of a mouse model of age-related hearing loss. Exp Gerontol 2014; 51: 8-14.

13. Someya S, Xu J, Kondo K, Ding D, Salvi RJ, Yamasoba T et al. Age-related hearing loss in C57BL/6J mice is mediated by Bak-dependent mitochondrial apoptosis. Proc Natl Acad Sci USA 2009; 106: 19432-19437.

14. Tan CC, Yu JT, Tan MS, Jiang T, Zhu XC, Tan L. Autophagy in aging and neurodegenerative diseases: implications for pathogenesis and therapy. Neurobiol Aging 2014; 35: 941-957.

15. Mizushima N, Levine B, Cuervo AM, Klionsky DJ. Autophagy fights disease through cellular self-digestion. Nature 2008; 451: 1069-1075.

16. Scherz-Shouval R, Elazar Z. Regulation of autophagy by ROS: physiology and pathology. Trends Biochem Sci 2011; 36: 30-38.

17. Levine B, Mizushima N, Virgin HW. Autophagy in immunity and inflammation. Nature 2011; 469: 323-335.

18. Giordano S, Darley-Usmar V, Zhang J. Autophagy as an essential cellular antioxidant pathway in neurodegenerative disease. Redox Biol 2014; 2: 82-90.

19. Kesidou E, Lagoudaki R, Touloumi O, Poulatsidou KN, Simeonidou C. Autophagy and neurodegenerative disorders. Neural Reg Res 2013; 8: 2275-2283.

20. Kwon JJ, Willy JA, Quirin KA, Wek RC, Korc M, Yin XM et al. Novel role of miR-29a in pancreatic cancer autophagy and its therapeutic potential. Oncotarget 2016; 44: 71635-71650.

21. Lynch-Day MA, Mao K, Wang K, Zhao M, Klionsky DJ. The role of autophagy in Parkinson's disease. Cold Spring Harb Perspect Med 2012; 2: a9357.

22. Nixon RA. The role of autophagy in neurodegenerative disease. Nat Med 2013; 19: 983-997.

23. Cheung ZH, Ip NY. Autophagy deregulation in neurodegenerative diseases - recent advances and future perspectives. J Neurochem 2011; 118: 317-325.

24. Youn CK, Kim J, Park JH, Do NY, Cho SI. Role of autophagy in cisplatin-induced ototoxicity. Int J Pediatr Otorhinolaryngol 2015; 79: 1814-1819.

25. Yuan H, Wang X, Hill K, Chen J, Lemasters J, Yang SM et al. Autophagy attenuates noise-induced hearing loss by reducing oxidative stress. Antioxid Redox Signal 2015; 22: 1308-1324.

26. Fang B, Xiao H. Rapamycin alleviates cisplatin-induced ototoxicity in vivo. Biochem Biophys Res Commun 2014; 448: 443-447.

27. Menardo J, Tang Y, Ladrech S, Lenoir M, Casas F, Michel $C$ et al. Oxidative stress, inflammation, and autophagic stress as the key mechanisms of premature age-related hearing loss in SAMP8 mouse cochlea. Antioxid Redox Signal 2012; 16: 263-274.

28. Bates DJ, Li N, Liang R, Sarojini H, An J, Masternak MM et al. MicroRNA regulation in Ames dwarf mouse liver may contribute to delayed aging. Aging Cell 2010; 9: 1-18.

29. Kato M, Slack FJ. microRNAs: small molecules with big roles $-C$. elegans to human cancer. Biol Cell 2008; 100: 71-81.

30. Zhang Q, Liu H, McGee J, Walsh EJ, Soukup GA, He DZ. Identifying microRNAs involved in degeneration of the organ of corti during age-related hearing loss. PLOS ONE 2013; 8: e62786.

31. Yang J, Chen D, He Y, Melendez A, Feng Z, Hong Q et al. MiR-34 modulates Caenorhabditis elegans lifespan via repressing the autophagy gene atg9. Age (Dordr) 2013; 35: 11-22.

32. Liu N, Landreh M, Cao K, Abe M, Hendriks GJ, Kennerdell JR et al. The microRNA miR-34 modulates ageing and neurodegeneration in Drosophila. Nature 2012; 482: 519-523.

33. Tan L, Yu JT, Tan L. Causes and consequences of microRNA dysregulation in neurodegenerative diseases. Mol Neurobiol 2015; 51: 1249-1262.

34. Rubinsztein DC, Cuervo AM, Ravikumar B, Sarkar S, Korolchuk V, Kaushik S et al. In search of an 'autophagomometer'. Autophagy 2009; 5: 585-589.

35. Klionsky D, Abdelmohsen K, Abe A, Abedin M, Abeliovich H, Acevedo Arozena A et al. Guidelines for the use and interpretation of assays for monitoring autophagy (3rd edition). Autophagy 2016; 12: 1-222.

36. Kalinec GM, Park C, Thein P, Kalinec F. Working with auditory HEI-OC1 cells. J Vis Exp 2016; 115: e54425, 10-3791.

37. Kalinec G, Thein P, Park C, Kalinec F. HEl-OC1 cells as a model for investigating drug cytotoxicity. Hear Res 2016; 335: 105-117.

38. Longatti A, Tooze SA. Vesicular trafficking and autophagosome formation. Cell Death Differ 2009; 16: 956-965.

39. Amaral JD, Castro RE, Sola S, Steer CJ, Rodrigues CM. p53 is a key molecular target of ursodeoxycholic acid in regulating apoptosis. J Biol Chem 2007; 282: 34250-34259.

40. Castro RE, Ferreira DM, Zhang $X$, Borralho PM, Sarver AL, Zeng $Y$ et al. Identification of microRNAs during rat liver regeneration after partial hepatectomy and modulation by ursodeoxycholic acid. Am J Physiol Gastrointest Liver Physiol 2010; 299: G887-G897.

41. Castro RE, Ferreira DM, Afonso MB, Borralho PM, Machado MV, Cortez-Pinto $\mathrm{H}$ et al. $\mathrm{miR}-34 \mathrm{a} / \mathrm{SIRT} 1 / \mathrm{p} 53$ is suppressed by ursodeoxycholic acid in the rat liver and activated by disease severity in human non-alcoholic fatty liver disease. J Hepatol 2013; 58: 119-125.

42. Tai $\mathrm{H}$, Wang Z, Gong H, Han X, Zhou J, Wang X et al. Autophagy impairment with lysosomal and mitochondrial dysfunction is an important characteristic of oxidative stress-induced senescence. Autophagy 2017; 13: 99-113.

43. Fujii S, Hara H, Araya J, Takasaka N, Kojima J, Ito S et al. Insufficient autophagy promotes bronchial epithelial cell senescence in chronic obstructive pulmonary disease. Oncolmmunology 2012; 1: 630-641. 
44. Kang HT, Lee KB, Kim SY, Choi HR, Park SC. Autophagy impairment induces premature senescence in primary human fibroblasts. PLOS ONE 2011; 6: e23367.

45. Morgado AL, Xavier JM, Dionisio PA, Ribeiro MF, Dias RB, Sebastiao AM et al. MicroRNA-34a modulates neural stem cell differentiation by regulating expression of synaptic and autophagic proteins. Mol Neurobiol 2015; 51: 1168-1183.

46. Huang J, Sun W, Huang $\mathrm{H}$, Ye J, Pan W, Zhong $Y$ et al. miR-34a modulates angiotensin IIinduced myocardial hypertrophy by direct inhibition of ATG9A expression and autophagic activity. PLOS ONE 2014; 9: e94382.

47. Puri C, Renna M, Bento CF, Moreau K, Rubinsztein DC. Diverse autophagosome membrane sources coalesce in recycling endosomes. Cell 2013; 6: 1285-1299.

48. Reggiori F, Tooze SA. Autophagy regulation through Atg9 traffic. J Cell Biol2012; 198: 151-153.

49. Webber JL, Young AR, Tooze SA. Atg9 trafficking in mammalian cells. Autophagy 2007; 3: 54-56.

50. Orsi A, Razi M, Dooley HC, Robinson D, Weston AE, Collinson LM et al. Dynamic and transient interactions of Atg9 with autophagosomes, but not membrane integration, are required for autophagy. Mol Biol Cell 2012; 23: 1860-1873.

51. Nezich CL, Wang C, Fogel Al, Youle RJ. MiT/TFE transcription factors are activated during mitophagy downstream of Parkin and Atg5. J Cell Biol 2015; 210: 435-450.

52. Yang Y, Cheng HW, Qiu Y, Dupee D, Noonan M, Lin YD et al. MicroRNA-34a plays a key role in cardiac repair and regeneration following myocardial infarction. Circ Res 2015; 117: 450-459.

53. Huang R, Xu Y, Wan W, Shou X, Qian J, You Z et al. Deacetylation of nuclear LC3 drives autophagy initiation under starvation. Mol Cell 2015; 57: 456-466.

54. Huang R, Liu W. Identifying an essential role of nuclear LC3 for autophagy. Autophagy 2015; 11: 852-853.

55. Abdelkader NF, Safar MM, Salem HA. Ursodeoxycholic acid ameliorates apoptotic cascade in the rotenone model of Parkinson's disease: modulation of mitochondrial perturbations. Mol Neurobiol 2016; 53: 810-817.
56. Cortez LM, Campeau J, Norman G, Kalayil M, Van der Merwe J, McKenzie D et al. Bile acids reduce prion conversion, reduce neuronal loss, and prolong male survival in models of prion disease. J Virol 2015; 89: 7660-7672.

57. Cortez L, Sim V. The therapeutic potential of chemical chaperones in protein folding diseases. Prion 2014; 8: 197-202.

58. Cao AL, Wang L, Chen X, Wang YM, Guo HJ, Chu S et al. Ursodeoxycholic acid and 4 phenylbutyrate prevent endoplasmic reticulum stress-induced podocyte apoptosis in diabetic nephropathy. Lab Invest 2016; 96: 610-622.

59. Rodriguez VA, Rivoira MA, Perez AV, Marchionatti AM, Tolosa DTN. Ursodeoxycholic and deoxycholic acids: differential effects on intestinal $\mathrm{Ca}(2+)$ uptake, apoptosis and autophagy of rat intestine. Arch Biochem Biophys 2016; 591: 28-34.

(i) Cell Death and Disease is an open-access journa published by Nature Publishing Group. This work is licensed under a Creative Commons Attribution 4.0 International License. The images or other third party material in this article are included in the article's Creative Commons license, unless indicated otherwise in the credit line; if the material is not included under the Creative Commons license, users will need to obtain permission from the license holder to reproduce the material. To view a copy of this license, visit http://creativecommons.org/licenses/by/4.0/

(C) The Author(s) 2017 